\title{
BJMHR
}

British Journal of Medical and Health Research

Journal home page: www.bjmhr.com

\section{Identification and Antibiotic Susceptibility Testing of Klebsiella species from various Clinical specimens in a tertiary care hospital}

Santosh kumar Gupta ${ }^{1 *}$, Suresh Jaiswal ${ }^{1}$, Bhoopendra Sharma ${ }^{1}$ \& Baijanti Mishra ${ }^{2}$

1.School of Health and Allied sciences, Faculty of Health Sciences, Pokhara University, Kaski, Nepal.

2.St. Johns Academy of Health Science, Banglore, Karnataka, India.

\section{ABSTRACT}

Klebsiella are Gram-negative, shorter and thicker rod shaped bacilli. On Mac-Conkey agar it shows mucoid colonies due to presence of polysaccharide capsule.Total 50 isolates of Klebsiella species collected from different clinical samples were studied biochemically. The different samples were urine 19 (38\%), pus 15 (30\%), blood 11 (22\%), and sputum 5 (10\%). Antibiotic susceptibility tests were done by Kirby Bauer disk diffusion method and the ESBL detection by double disc synergy method.Klebsiellapneumoniae were the most common 48 $(96 \%)$, followed by Klebsiellaoxytoca $2(4 \%)$ in our study. Antibiotic resistance to ampicillin were $100 \%$, percentages to cephalosporins were high: cefozolin and cefuroxime $30(60 \%)$, cefotaxime 27 (54\%) and cefoperazone and ceftazidime were 28 (56\%). Most of those 21 (42\%) were from the infection associated in the hospital. Resistance to Aminoglycosides ranged from 60 to $70 \%$. Resistance to co-trimoxazole and tetracycline were 25 (50\%) and 27 (54\%) respectively. Resistance to fluoroquinolones were 28 (56\%). Resistance to carbapenem (meropenem) were $20(40 \%)$ and to piperacillin sulbactam were 21 (42\%). Resistance to third generation cephalosporins were $15(30 \%)$ out of which $9(60 \%)$ showed ESBL production using double disc synergy method

Keywords: Urinary tract infection, Community acquired pneumonia, ESBL, AST

*Corresponding Author Email: skgupta7124@gmail.com Received 15 July 2018, Accepted 22 July 2018 


\section{INTRODUCTION}

Antimicrobial resistance has become now days a serious public health problem worldwide. Infections caused by resistant bacteria have increased morbidity and mortality than those caused by susceptible pathogens ${ }^{1,2}$. Infections caused by resistant bacteria led to prolonged hospital stays, increased health care costs and in many cases to untreatable infections ${ }^{3}$. Klebsiella has a prominent capsule which is responsible for the mucoid appearance of the isolated colonies and enhances the virulence of the organism in-vivo. Klebsiella pneumoniae is the most important Klebsiella species from a medical stand point and most commonly isolated from the clinical specimens. Pneumonia caused by Klebsiella is frequently associated with necrotic destruction of the alveolar space, causing cavity formation and production of blood stained sputum ${ }^{4}$.It is also associated with long term care facilities causing nosocomial infections. Klebsiella also cause wound, soft tissue infection and urinary tract infection.

Beta-lactams antibiotics are the most frequently prescribed antibiotics. Emerging resistance to these antibiotics among gram negative bacilli limited their utility. Extended spectrum [beta]-lactamases (ESBL) producing Klebsiella spp. has been frequently implicated in outbreaks in intensive care units and neonatal intensive care units (NICUs) ${ }^{5}$. Klebsiella pneumonia are ubiquitously present and reported worldwide. In recent years, $K$. pneumonia has become important pathogens in nosocomial infections. The importance of $K$. pneumonia species in the ever increasing number of gram negative aerobic bacillary nosocomial infections in the United States ${ }^{6}$ and India ${ }^{7}$ has been well documented. Over 100 variations and point mutations in TEM gene were reported by DNA sequencing. These mutations are the most responsible factor for resistance to beta lactams in these isolates. [The SHV family of [beta]-lactamases has been derived from Klebsiella spp. SHV-1 is universally found in $K$. pneumoniae, evolved as a chromosomal gene in Klebsiella spp. and was later incorporated into a plasmid, which has spread to other entero-bacterial species. ${ }^{8}$. A total of 40 types of SHV type ESBL enzymes are already reported.

There are various reports on the prevalence of ESBL producing Klebsiella spp. from India. A recent study by Khan Erum et al describes occurrence of genetic variants in $K$. pneumoniae from clinical samples of various origin and reported that isolates having both TEM and SHV genes were more common than TEM and SHV alone.

Epidemic strains of cephalosporin resistant $K$. pneumoniae have been associated with increased morbidity and mortality in hospitalized patients ${ }^{9}$. However, most Klebsiellae infections now occur in the hospital. Antibiotic - resistant strains have been responsible for a number of outbreaks of nosocomial infections in ICUS and neonatal nurseries .The most common clinical syndromes are pneumonia, UTI, abdominal infection, surgical site infection, 
soft tissue infection and subsequent bacteremia. K. pneumoniae subspecies rhinoscleromatis is the causative agent of rhinoscleroma - a granulomatous, slowly progressive (over months to years) infection of the upper respiratory mucosa that causes necrosis and occasional obstruction of the nasal passages. K.pneumoniae subspecies ozenae has been implicated in chronic atrophic rhinitis and in rare cases of invasive disease in compromised hosts.

Hence, the present study was undertaken to isolate and identify Klebsiella spp. from various clinical samples and to know their antibiotic susceptibility pattern in an urban tertiary care centre.

\section{MATERIALS AND METHOD:}

A total of 50 isolates of Klebsiella species from various clinical samples received in the department of microbiology St. Johns medical college and hospital (SJMCH) were studied during the period Jan 2010 to Dec. 2010. The strains were sub cultured on MacConkey agar to obtain pure growth. Subsequently each isolate were preserved in nutrient agar deeps for further studies. Presumptively identified Klebsiella species with significant growth and pure culture were included while Klebsiella species with insignificant /mixed growth were excluded,

The presumptively identified Klebsiella species isolated from various clinical samples, biochemically were identified to the genus and species level as per the recommended standards. Antibiotic susceptibility testing of the isolates was performed by Kirby-Bauer disc diffusion method. The antibiotic concentration of disc used and zone size interpretation was in accordance with clinical laboratory standards institute (CLSI) ${ }^{10}$ Different antibiotics used were as Ampicillin, Piperacillin, Cefazolin, Cefuroxime, Cefotaxime, Cefaperazone, Cetazidime, Gentamicin, Netilmycin, Amikacin, Cotrimoxazole, Tetracycline, Ciprofloxacin, Meropenem and Piperacillin + Tazobactam

Muller Hinton agar media was prepared, autoclaved, allowed to cool in $50^{\circ} \mathrm{C} \&$ poured on to Petri dishes to give a uniform depth of $4 \mathrm{~mm}$. 3-5 colonies of strain was inoculated into $2 \mathrm{ml}$ peptone water and incubated at $37^{\circ} \mathrm{C}$ for $4 \mathrm{hrs}$. Then turbidity was adjusted to $0.5 \mathrm{Mac}$ farlandstandard $(1.5 \mathrm{X} 10 \mathrm{cfu} / \mathrm{m})$ with sterile normal saline.

Plates were inoculated within 15 min of preparation of the suspension so that the density did not change .A sterile cotton wool swab was dipped in to the suspension and surplus removed by rotation of the swab against the side of the tube above the fluid level .The medium was inoculated by even streaking of the swab over the entire surface of the plates in three directions. After the inoculums had dried, discs were applied with forceps and pressed gently to ensure even contact with the medium.

\section{Controls:}


ATCC Escherichia coli 25922 (American type culture collection) was used

\section{ESBL DETECTION:}

The strains found to be resistant to $3^{\text {rd }}$ generation cephalosporins were done ESBL detection.

\section{Method Used:}

Double disc synergy method ${ }^{11}$.

\section{PROCEDURE:}

All the strains screened for resistance to third generation cephalosporins using three discs cefotaxime, ceftriaxone and ceftazidime by Kirby Bauer disc diffusion method. Those strains showing resistance to at least two of them (zone of inhibition less than $18 \mathrm{~mm}$ ) were tested for ESBL in duplicate by the Kirby Bauer dic diffusion in Mueller Hinton agar plates by Jarlier technique. In this technique, susceptibility disk containing amoxicillin and clavulanic acid was placed at the centre and cefotaxime, ceftriaxone and ceftazidime disks were placed at $15 \mathrm{~mm}$ distance (centre to centre) on a lawn culture of the organism using turbidity standard of $0.5 \mathrm{McFarland}$. An increased zone of inhibition towards the clavulanic acid disk was taken as positive for ESBL.

\section{RESULTS AND DISCUSSION}

Klebsiella pneumoniae were the most common 48 (96\%), followed by Klebsiella oxytoca 2 (4\%) in our study. Antibiotic resistance to ampicillin were $100 \%$, percentages to cephalosporins were high: cefozolin and cefuroxime 30 (60\%), cefotaxime $27(54 \%)$ and cefoperazone and ceftazidime were $28(56 \%)$. Most of those $21(42 \%)$ were from the infection associated in the hospital. Resistance to Aminoglycosides ranged from 60 to $70 \%$. Resistance to co-trimoxazole and tetracycline were $25(50 \%)$ and 27 (54\%) respectively. Resistance to fluoroquinolones were 28 (56\%). Resistance to carbapenem (meropenem) were $20(40 \%)$ and to piperacillin -sulbactam were $21(42 \%)$.

Resistance to third generation cephalosporins were 15 (30\%) out of which 9 (60\%) showed ESBL production using double disc synergy method. Confirmation of ESBL production in all those strains using molecular method could not be done due to lack of infra-structure. 

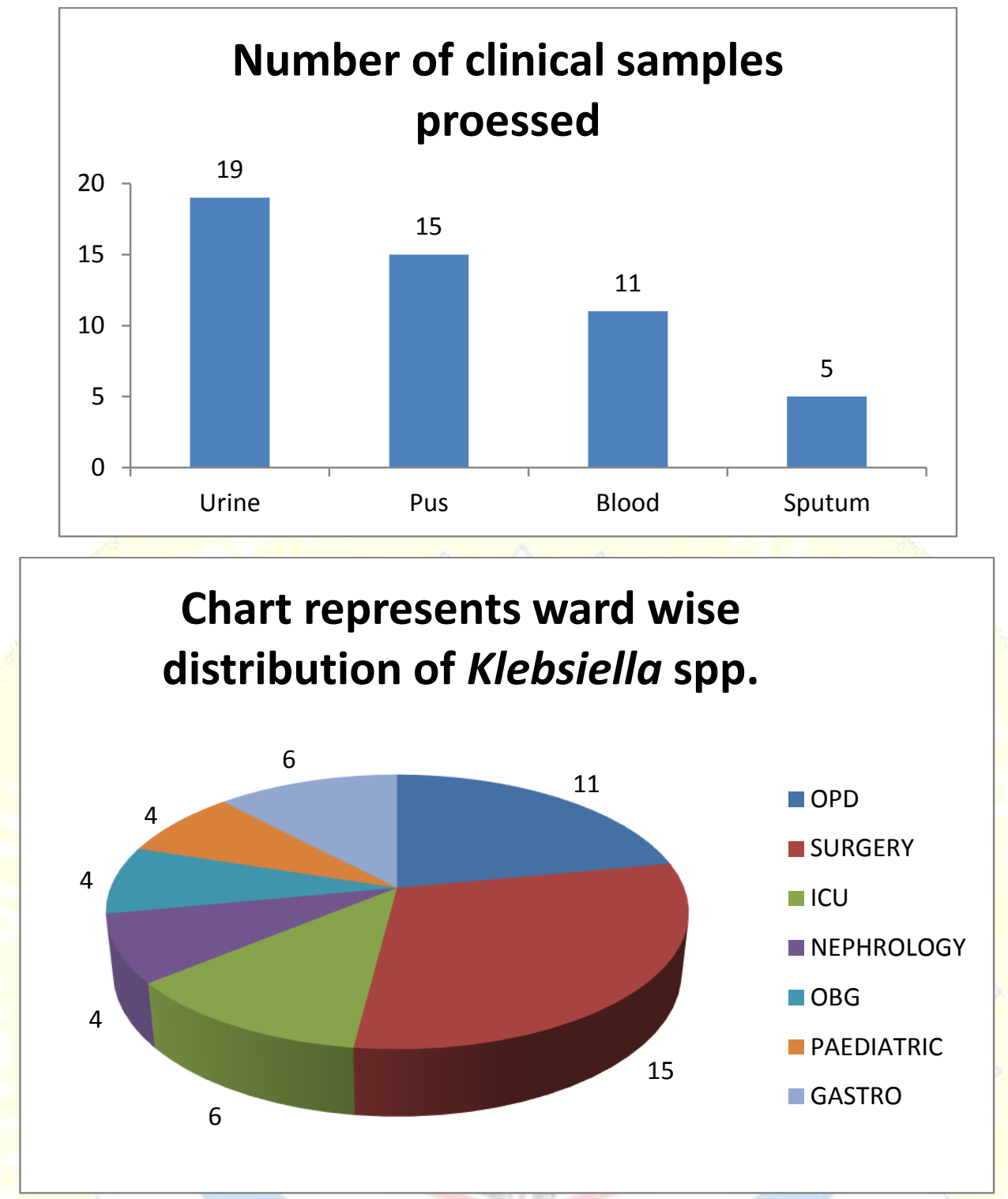

\begin{tabular}{lll}
\hline Antibiotics & No. Of isolates found resistance & Percentage of resistance \\
\hline Ampicillin & 50 & 100 \\
Piperacillin & 17 & 34 \\
Cefazolin & 30 & 60 \\
Cefuroxime & 30 & 60 \\
Cefotaxime & 27 & 54 \\
Cefaperazone & 28 & 56 \\
Ceftazidime & 28 & 56 \\
Gentamicin & 30 & 60 \\
Netilmycin & 35 & 70 \\
Amikacin & 30 & 60 \\
Cotrimoxazole & 25 & 50 \\
Tetracycline & 27 & 54 \\
Ciprofloxacin & 28 & 56 \\
Meropenem & 20 & 40 \\
Zosyn & 21 & 42 \\
\hline
\end{tabular}

Total Number Of Isolates Resistant To All 2 Or 3 Cephalosporin=15

ESBL DETECTION: 


\section{METHOD USED: double disc synergy method.}

\section{ESBL was detected in 9/15 strains (60\%)}

Klebsiella pneumoniae is considered an important pathogen causing nosocomial and community-acquired infections. It is often associated with the production of extended spectrum $\beta$-lactamases (ESBL) belonging to SHV and CTX-M families. These genes are frequently described as a part of complex integrons facilitating their horizontal transfer to other related as well as unrelated microbes. The present study was undertaken for the identification and antibiotic susceptibility testing of Klebsiella spp. from various specimens in a tertiary care hospital. ESBL producing isolates of Klebsiella pneumonia have been reported from various hospitals in India and other countries ${ }^{12}$. The need for ESBL production is under challenge to set the break point for injectable cephalosporins and aztreonam that accurately discriminate which ESBL-producing isolates can and cannot be reliably treated with these drugs.

The majority of our isolates were acquired from urine(38\%), followed by pus (30\%), then blood (22\%) and sputum (10\%) signifying the true infection. 6 isolates $(12 \%)$ were from the ICU putting them at high risk for ventilator associated pneumonia and 10?(\%) showed bacteremia with gram negative rods. 4 ? $(\%)$ cases showed community acquired bronchopneumonia associated with chronic obstructive lesions. Out of 50 strains, 48 (96\%) were due to the Klebsiella pneumoniae and 2 strains were $K$. oxytoca. Both those $K$. oxytoca strains were isolated as nosocomial pathogens, one from the pus wound and the other from the urine. Both the strains though similar to the K. pneumoniae biochemically, but formed indole and fermented dulcitol.

\section{CONCLUSION:}

Klebsiella isolates have been steadily increasing over the past years and they have been important source of transferable drug resistance. Indiscriminate use of third generation cephalosporins to treat gram negative bacterial infections is partly responsible for the emergence of resistance to beta-lactam antibiotics. Strict adherence to the hospital antibiotic policy and good infection control practices can play a significant role in reducing the increasing drug resistance .Epidemiological surveillance studies should provide useful information base to guide practice and policies on rational use of anti-infective agents and to eradicate the source of environmental.

\section{ACKNOWLEDGEMENT:}

The authors like to thank to the staff and administration of St. johns medical college and hospital for providing facility to carry out this research. 


\section{REFERENCES.}

1. Helms M, Vastrup P, Gerner-Smidt P, MølbakK.Excess mortality associated with Antimicrobial drug resistant Salmonella typhimurium.Emerg Infect Dis,2002; $8(5): 490-495$.

2. Travers K, Barza M; Morbidity of infections caused by antimicrobial-resistant bacteria.Clin Infect Dis, 2002; 34(3):S131-4.

3. Byarugaba DK; A view on antimicrobial resistance in developing countries and responsible risk factors. Int J Antimicrob Agents, 2004; 24(2):105-110.

4. Murrary P R , Kobayashi G S , P faller M A, Rosenthal K S. Medical microbiology. 2nd. Mosby; 1994.page no-239.

5. Pawa AK, Ramji S, Prakash K, Thirupuram S. Neonatal nosocomial infection. Profile and risk factors. Indian paediatrics 1997;34:297-302.

6. Graybill JR, Marshall LW, Charache P, Wallace CK,Melwin VK; Nosocomial pneumonia: A continuing major problem. Am.Rev.Respir.Dis. 1973; 108:1130-1140.

7. Mathur NB, Khalib A, Sarkar R, Puri RK; Mortality in neonatal septicaemia with involvement of mother in management. Ind. J. Pediatri.1991,; 28 (2) :1259-1264.

8. Jacoby GA and Silvia L (2005) Mechanisms of Disease: The new $\beta$ lactamases. N Engl J Med 352:380-391.

9. Dean AD, Dean AJ, Burton AH, Dicker RC; Epi- programme for epidemiology on microcomputers.VSD. Inc Stone Mountain Ga, 1990.

10. Clinical laboratory standards institute performance for anti microbial disk susceptibility tests 8th ed. Approved standards M2- A8 wayne, pa; Clinical Laboratory standards 2003.

11. National Committee for clinical laboratory standards, 1998, performance standards for antimicrobial disk susceptibility testing, Eight informational supplement, M-10058, National Committee for clinical laboratory standards, Wayne Pa.

12. P. Datta, A. Thakur, B. Mishra. Prevalence of Clinical strains resistant to various beta-lactams in a tertiary care hospital in India. Ind J Med Microbiol 2004; 57:146-9.

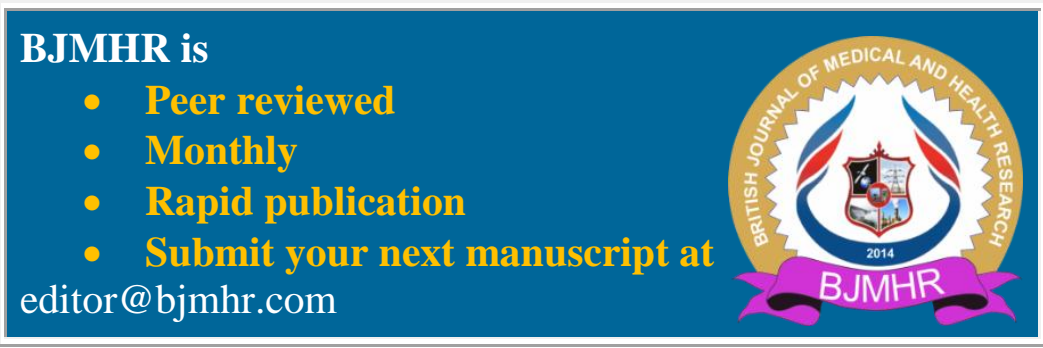

